\title{
A new extended discrete KP hierarchy and generalized dressing method
}

\author{
Yuqin Yao ${ }^{1} \sqrt{1}$, Xiaojun Liu $2 \sqrt{2}$ and Yunbo Zeng $\sqrt{3}$ \\ ${ }^{1)}$ Department of Mathematical Science, Tsinghua University, Beijing, 100084, PR \\ China \\ ${ }^{2)}$ Department of Applied Mathematics, China Agricultural University, Beijing, \\ 100083, PR China
}

\begin{abstract}
Inspired by the squared eigenfunction symmetry constraint, we introduce a new $\tau_{k}$-flow by "extending" a specific $t_{n}$-flow of discrete KP hierarchy $(\mathrm{DKPH})$. We construct extended discrete KPH (exDKPH), which consists of $t_{n^{-}}$ flow, $\tau_{k}$-flow and $t_{n}$ evolution of eigenfunction and adjoint eigenfunctions, and its Lax representation. The exDKPH contains two types of discrete KP equation with self-consistent sources (DKPESCS). Two reductions of exDKPH are obtained. The generalized dressing approach for solving the exDKPH is proposed and the N-soliton solutions of two types of the DKPESCS are presented.
\end{abstract}

\section{Introduction}

Generalizations of soliton hierarchy attract a lot of interests from both physical and mathematical points and there were some methods to generalize the soliton hierarchy 1-4. Recently, a systematic approach inspired by squared eigenfunction symmetry constraint was proposed to construct the extended KP hierarchy [5]. By this method, the extended two-dimensional Toda lattice hierarchy, the extended CKP hierarchy and the extended q-deformed KP hierarchy have been obtained [6]- 8].

The discrete KP hierarchy(DKPH) [9]-12] is an interesting object in the research of the discrete integrable systems and the discretization of the integrable systems 13. The Sato's approach for the discrete KPH was presented in 11. Naturally, there are some similar properties between discrete KPH and $\mathrm{KPH}[14$, such as tau function[12, 14, Hamiltonian structure 12] and gauge transformation [10, 15, 16, etc. In [10, Oevel has given explicitly two types of gauge transformation operators of the discrete KPH. In [16, the combined gauge operator and the determinant representation of the operator have been obtained.

In this paper, we will construct the extension of the discrete $\mathrm{KPH}(\operatorname{exDKPH})$. Inspired by the squared eigenfunction symmetry constraint of discrete KP hierarchy [10, we introduced the new $\tau_{k}$-flow by "extending" a specific $t_{n}$-flow of discrete KP hierarchy. Then we find the exDKPH consisting of $t_{n}$-flow of

\footnotetext{
1yqyao@math.tsinghua.edu.cn

2 tigertooth4@gmail.com

${ }^{3}$ Corresponding author:

yzeng@math.tsinghua.edu.cn
} 
discrete KP hierarchy, $\tau_{k}$-flow and the $t_{n}$-evolutions of eigenfunctions and adjoint eigenfunctions. The commutativity of $t_{n}$-flow and $\tau_{k}$-flow gives rise to zero curvature representation for exDKPH. Also the Lax representation of exDKPH is derived. Due to the introduction of $\tau_{k}$-flow the exDKPH contains two time series $\left\{t_{n}\right\}$ and $\left\{\tau_{k}\right\}$ and more components by adding eigenfunctions and adjoint eigenfunctions. The exDKPH contains the first type and second type of discrete KP equation with self-consistent sources(DKPESCS). The KP equation with self-consistent sources arose in some physical models describing the interaction of long and short waves [4. The similarity of KP equation and discrete $\mathrm{KP}$ equation enables us to speculate on the potential application of discrete $\mathrm{KP}$ equation with self-consistent sources. By $t_{n}$-reduction and $\tau_{k}$-reduction, the exDKPH reduces to a discrete 1+1-dimensional integrable hierarchy with self-consistent sources and constrained discrete KP hierarchy, respectively.

The dressing method is an important tool for solving soliton hierarchy [12]. However this method can not be applied directly for solving the "extended" hierarchy. A generalized dressing approach for exKPH is proposed in [17. In this paper, with the combination of dressing method and variation of constants method, a generalization to the dressing method for exDKPH is presented, which is based on the dressing method for discrete KPH [11] and the similar approach for finding Wronskian solutions to constrained KP hierarchy [18. In this way, we can solve the entire hierarchy of exDKPH in an unified and simple manner. As the special cases, the N-soliton solutions of the both types of DKPESCS are obtained simultaneously.

This paper will be organized as follows. In Sec.2, we present the exDKPH and its Lax pair, which includes two types of DKPESCS. In Sec.3, $t_{n}$-reduction and $\tau_{k}$ - reduction for the exDKPH are given. In Sec.4, we discuss the generalized dressing method for the exDKPH. In Sec.5, we present the N-soliton solutions of the DKPESCS.

\section{New extended discrete KP hierarchy}

We denote the shift and the difference operators acting on the associative ring $F$ of functions by $\Gamma$ and $\Delta$, respectively, as follows

$$
\begin{gathered}
F=\left\{f(l)=f\left(l, t_{1}, t_{2}, \cdots, t_{i}, \cdots\right) ; l \in \mathbb{Z}, t_{i} \in \mathbb{R}\right\} \\
\Gamma(f(l))=f(l+1)=f^{(1)}(l), \Delta(f(l))=f(l+1)-f(l) .
\end{gathered}
$$

In this paper, we use $P(f)$ to denote an action of difference operator $P$ on the function $f$, while $P f$ means the multiplication of difference operator $P$ and zero order difference operator $f$. Define the following operation

$$
\Delta^{j} f=\sum_{i=0}^{\infty}\left(\begin{array}{c}
j \\
i
\end{array}\right)\left(\Delta^{i}(f(l+j-i))\right) \Delta^{j-i}, \quad\left(\begin{array}{c}
j \\
i
\end{array}\right)=\frac{j(j-1) \cdots(j-i+1)}{i !} .
$$

Also, we define the adjoint operator to the $\Delta$ operator by $\Delta^{*}$

$$
\Delta^{*}(f(l))=\left(\Gamma^{-1}-I\right)(f(l))=f(l-1)-f(l),
$$




$$
\Delta^{* j} f=\sum_{i=0}^{\infty}\left(\begin{array}{l}
j \\
i
\end{array}\right)\left(\Delta^{* i}(f(l+i-j))\right) \Delta^{* j-i} .
$$

Let $P=\sum_{j=-\infty}^{k} f_{j}(l) \Delta^{j}$, the adjoint operator $P^{*}$ is defined by $P^{*}=\sum_{j=-\infty}^{k} \Delta^{* j} f_{j}(l)$.

The Lax equation of the DKP hierarchy is given by $[9,11$

$$
L_{t_{n}}=\left[B_{n}, L\right],
$$

where $L=\Delta+f_{0}+f_{1} \Delta^{-1}+f_{2} \Delta^{-2}+\cdots$ is a pseudo-difference operator with potential functions $f_{i} \in F, B_{n}=L_{+}^{n}$ stands for the difference part of $L^{n}$. The commutativity of $t_{n^{-}}$and $t_{m}$-flow gives rise to the zero-curvature equations for DKP hierarchy:

$$
B_{n, t_{m}}-B_{m, t_{n}}+\left[B_{n}, B_{m}\right]=0 .
$$

with the Lax pair given by

$$
\psi_{t_{n}}=B_{n}(\psi), \quad \psi_{t_{m}}=B_{m}(\psi) .
$$

The $t_{n}$ evolutions of eigenfunction $\psi$ and adjoint eigenfunction $\phi$ read

$$
\psi_{t_{n}}=B_{n}(\psi), \quad \phi_{t_{n}}=-B_{n}^{*}(\phi) .
$$

For $n=2, m=1$, (5) gives rise to the DKP equation 9 ]

$$
\Delta\left(f_{0 t_{2}}+2 f_{0 t_{1}}-2 f_{0} f_{0 t_{1}}\right)=(\Delta+2) f_{0 t_{1} t_{1}} .
$$

It is known that the squared eigenfunction symmetry constraint given by 10 ]

$$
\begin{gathered}
\tilde{B}_{k}=B_{k}+\sum_{i=1}^{N} \psi_{i} \Delta^{-1} \phi_{i} \\
\psi_{i, t_{n}}=B_{n}\left(\psi_{i}\right), \phi_{i, t_{n}}=-B_{n}^{*}\left(\phi_{i}\right), i=1, \cdots, N,
\end{gathered}
$$

is compatible with DKP hierarchy. Here $N$ is an arbitrary natural number, $\psi_{i}$ and $\phi_{i}$ are $N$ different eigenfunctions and adjoint eigenfunctions of the equations (9c). This compatibility enables us to construct a new extended discrete KP hierarchy (exDKPH) as

$$
\begin{aligned}
L_{t_{n}} & =\left[B_{n}, L\right], \\
L_{\tau_{k}} & =\left[B_{k}+\sum_{i=1}^{N} \psi_{i} \Delta^{-1} \phi_{i}, L\right], \\
\psi_{i, t_{n}} & =B_{n}\left(\psi_{i}\right), \phi_{i, t_{n}}=-B_{n}^{*}\left(\phi_{i}\right), i=1, \cdots, N .
\end{aligned}
$$

We have the following lemma.

Lemma 1. Let $Q=a \Delta^{k}, k \geq 1$, then

$$
\begin{aligned}
\left(\Delta^{-1} \phi Q\right)_{-} & =\Delta^{-1} Q^{*}(\phi) \\
{\left[B_{n}, \psi \Delta^{-1} \phi\right]_{-} } & =B_{n}(\psi) \Delta^{-1} \phi-\psi \Delta^{-1} B_{n}^{*}(\phi) .
\end{aligned}
$$


Proof. Using $f \Delta=\Delta \Gamma^{-1}(f)-\Delta\left(\Gamma^{-1}(f)\right), \Delta^{*}=-\Delta \Gamma^{-1}$, we have

$$
\begin{gathered}
\left(\Delta^{-1} \phi a \Delta^{k}\right)_{-}=\left(\Delta^{-1} \Delta \Gamma^{-1}(\phi a) \Delta^{k-1}-\Delta^{-1} \Delta\left(\Gamma^{-1}(\phi a)\right) \Delta^{k-1}\right)_{-} \\
=-\left(\Delta^{-1} \Delta\left(\Gamma^{-1}(\phi a)\right) \Delta^{k-1}\right)_{-}=\cdots \\
=(-1)^{k} \Delta^{-1} \Delta^{k}\left(\Gamma^{-k}(\phi a)\right)=\Delta^{-1} \Delta^{* k}(\phi a)=\Delta^{-1} Q^{*}(\phi)
\end{gathered}
$$

which yields to (10a) and (10b).

Proposition 1. The commutativity of (9a) and (9b) under (9c) gives rise to the following zero-curvature representation for exDKPH (9)

$$
\begin{gathered}
B_{n, \tau_{k}}-\left(B_{k}+\sum_{i=1}^{N} \psi_{i} \Delta^{-1} \phi_{i}\right)_{t_{n}}+\left[B_{n}, B_{k}+\sum_{i=1}^{N} \psi_{i} \Delta^{-1} \phi_{i}\right]=0 \\
\psi_{i, t_{n}}=B_{n}\left(\psi_{i}\right), \phi_{i, t_{n}}=-B_{n}^{*}\left(\phi_{i}\right), \quad i=1,2, \cdots, N
\end{gathered}
$$

with the Lax representation given by

$$
\Psi_{t_{n}}=B_{n}(\Psi), \quad \Psi_{\tau_{k}}=\left(B_{k}+\sum_{i=1}^{N} \psi_{i} \Delta^{-1} \phi_{i}\right)(\Psi) .
$$

Proof. For convenience, we omit $\sum$. By (9) and Lemma 1, we have

$$
\begin{aligned}
& B_{n, \tau_{k}}=\left(L_{\tau_{k}}^{n}\right)_{+}=\left[B_{k}+\psi \Delta^{-1} \phi, L^{n}\right]_{+}=\left[B_{k}+\psi \Delta^{-1} \phi, L_{+}^{n}\right]_{+}+\left[B_{k}+\psi \Delta^{-1} \phi, L_{-}^{n}\right]_{+} \\
& =\left[B_{k}+\psi \Delta^{-1} \phi, L_{+}^{n}\right]-\left[B_{k}+\psi \Delta^{-1} \phi, L_{+}^{n}\right]_{-}+\left[B_{k}, L_{-}^{n}\right]_{+}=\left[B_{k}+\psi \Delta^{-1} \phi, L_{+}^{n}\right] \\
& -\left[\psi \Delta^{-1} \phi, B_{n}\right]_{-}+\left[B_{n}, L^{k}\right]_{+}=\left[B_{k}+\psi \Delta^{-1} \phi, B_{n}\right]+\left(B_{k}+\psi \Delta^{-1} \phi\right)_{t_{n}} .
\end{aligned}
$$

Remark. The exDKPH (11) extends the DKPH (5) by containing two time series $\left\{t_{n}\right\}$ and $\left\{\tau_{k}\right\}$ and more components $\psi_{i}$ and $\phi_{i}, i=1, \cdots, N$.

Example 1. The first type of DKPSCS is given by (11) with $n=1, k=2$

$$
\begin{aligned}
& \Delta\left(f_{0 \tau_{2}}+2 f_{0 t_{1}}-2 f_{0} f_{0 t_{1}}\right)=(\Delta+2) f_{0 t_{1} t_{1}}-\Delta^{2} \sum_{i=1}^{N}\left(\psi_{i} \phi_{i}^{(-1)}\right), \\
& \psi_{i, t_{1}}=\Delta\left(\psi_{i}\right)+f_{0} \psi_{i}, \phi_{i, t_{1}}=-\Delta^{*}\left(\phi_{i}\right)-f_{0} \phi_{i}, i=1,2, \cdots, N .
\end{aligned}
$$

Its Lax representation is

$$
\begin{aligned}
& \Psi_{t_{1}}=\left(\Delta+f_{0}\right)(\Psi) \\
& \Psi_{\tau_{2}}=\left(\Delta^{2}+\left(f_{0}+f_{0}^{(1)}\right) \Delta+\Delta\left(f_{0}\right)+f_{1}^{(1)}+f_{1}+f_{0}^{2}+\sum_{i=1}^{N} \psi_{i} \Delta^{-1} \phi_{i}\right)(\Psi) .
\end{aligned}
$$


Example 2. The second type of DKPSCS is given by (11) with $n=2, k=1$

$$
\begin{gathered}
\Delta\left(f_{0 t_{2}}+2 f_{0 \tau_{1}}-2 f_{0} f_{0 \tau_{1}}\right)=(\Delta+2) f_{0 \tau_{1} \tau_{1}}+\sum_{i=1}^{N}\left[\Delta^{2}\left(\left(f_{0}+f_{0}^{-1}-2\right) \psi_{i} \phi_{i}^{-1}\right)\right. \\
\left.+\Delta\left(\psi_{i}^{(2)} \phi_{i}-\psi_{i} \phi_{i}^{(-2)}\right)+\Delta\left((\Gamma+1)\left(\psi_{i} \phi_{i}^{(-1)}\right)_{\tau_{1}}\right)\right] \\
\psi_{i, t_{2}}=\Delta^{2}\left(\psi_{i}\right)+\left(f_{0}+f_{0}^{(1)}\right) \Delta\left(\psi_{i}\right)+\left(\Delta\left(f_{0}\right)+f_{1}^{(1)}+f_{1}+f_{0}^{2}\right) \psi_{i} \\
\phi_{i, t_{2}}=-\Delta^{* 2}\left(\psi_{i}\right)-\Delta^{*}\left(\left(f_{0}+f_{0}^{(1)}\right) \psi_{i}\right)-\left(\Delta\left(f_{0}\right)+f_{1}^{(1)}+f_{1}+f_{0}^{2}\right) \psi_{i}
\end{gathered}
$$

Its Lax representation is

$$
\begin{aligned}
& \Psi_{t_{2}}=\left(\Delta^{2}+\left(f_{0}+f_{0}^{(1)}\right) \Delta+\Delta\left(f_{0}\right)+f_{1}^{(1)}+f_{1}+f_{0}^{2}\right)(\Psi) \\
& \Psi_{\tau_{1}}=\left(\Delta+f_{0}+\sum_{i=1}^{N} \psi_{i} \Delta^{-1} \phi_{i}\right)(\Psi) .
\end{aligned}
$$

\section{Reductions of the exDKPH}

\subsection{The $t_{n}$ - reduction}

The $t_{n}$-reduction is given by

$$
L^{n}=B_{n} \quad \text { or } \quad L_{-}^{n}=0 .
$$

Then we have

$$
\left(L^{n}\right)_{t_{n}}=\left[B_{n}, L^{n}\right]=0, \quad B_{n, t_{n}}=0 .
$$

So $L$ is independent of $t_{n}$ and we have

$$
B_{n}\left(\psi_{i}\right)=L^{n}\left(\psi_{i}\right)=\lambda_{i}^{n} \psi_{i}, B_{n}^{*}\left(\phi_{i}\right)=\lambda_{i}^{n} \phi_{i} .
$$

Then we can drop $t_{n}$ dependency from (11) and obtain

$$
\begin{aligned}
B_{n, \tau_{k}} & =\left[\left(B_{n}\right)_{+}^{\frac{k}{n}}+\sum_{i=1}^{N} \psi_{i} \Delta^{-1} \phi_{i}, B_{n}\right], \\
B_{n}\left(\psi_{i}\right) & =\lambda_{i}^{n} \psi_{i}, B_{n}^{*}\left(\phi_{i}\right)=\lambda_{i}^{n} \phi_{i}, i=1,2, \cdots, N,
\end{aligned}
$$

with the Lax pair given by

$$
\Psi_{\tau_{k}}=\left(\left(B_{n}\right)_{+}^{\frac{k}{n}}+\sum_{i=1}^{N} \psi_{i} \Delta^{-1} \phi_{i}\right)(\Psi), B_{n}(\Psi)=\lambda^{n} \Psi .
$$


(19) can be regarded as discrete $(1+1)$-dimensional integrable hierarchy with self-consistent sources. When $n=2, k=1$, (19) gives rise to

$$
\begin{gathered}
2 \Delta\left(f_{0 \tau_{1}}-f_{0} f_{0 \tau_{1}}\right)=(\Delta+2) f_{0 \tau_{1} \tau_{1}}+\sum_{i=1}^{N}\left[\Delta^{2}\left(f_{0}+f_{0}^{(-1)}-2\right) \psi_{i} \phi_{i}^{-1}\right. \\
\left.+\Delta\left(\psi_{i}^{(2)} \phi_{i}-\psi_{i} \phi_{i}^{(-2)}\right)+\Delta(\Gamma+1)\left(\psi_{i} \phi_{i}^{(-1)}\right)_{\tau_{1}}\right] \\
\Delta^{2}\left(\psi_{i}\right)+\left(f_{0}+f_{0}^{(1)}\right) \Delta\left(\psi_{i}\right)+\left(\Delta\left(f_{0}\right)+f_{1}^{(1)}+f_{1}+f_{0}^{2}\right) \psi_{i}=\lambda_{i}^{2} \psi_{i}, \\
\Delta^{* 2}\left(\psi_{i}\right)+\Delta^{*}\left(\left(f_{0}+f_{0}^{(1)}\right) \psi_{i}\right)+\left(\Delta\left(f_{0}\right)+f_{1}^{(1)}+f_{1}+f_{0}^{2}\right) \psi_{i}=\lambda_{i}^{2} \phi_{i},
\end{gathered}
$$

which can be transformed to the first type of Veselov-Shabat equation [19] with self-consistent sources (VSESCS).

\subsection{The $\tau_{k}$ - reduction}

The $\tau_{k}$-reduction is given by [10]

$$
L^{k}=B_{k}+\sum_{i=1}^{N} \psi_{i} \Delta^{-1} \phi_{i} .
$$

By dropping $\tau_{k}$ dependency from (11), we obtain

$$
\begin{aligned}
& \left(B_{k}+\sum_{i=1}^{N} \psi_{i} \Delta^{-1} \phi_{i}\right)_{t_{n}}=\left[\left(B_{k}+\sum_{i=1}^{N} \psi_{i} \Delta^{-1} \phi_{i}\right)_{+}^{\frac{n}{k}}, B_{k}+\sum_{i=1}^{N} \psi_{i} \Delta^{-1} \phi_{i}\right], \\
& \psi_{i, t_{n}}=\left(B_{k}+\sum_{i=1}^{N} \psi_{i} \Delta^{-1} \phi_{i}\right)_{+}^{\frac{n}{k}}\left(\psi_{i}\right), \\
& \phi_{i, t_{n}}=-\left(B_{k}+\sum_{i=1}^{N} \psi_{i} \Delta^{-1} \phi_{i}\right)_{+}^{\frac{n}{k} *}\left(\phi_{i}\right), i=1,2, \cdots, N,
\end{aligned}
$$

which is the k-constrained DKP hierarchy. When $n=1, k=2$, (21) leads to

$$
\begin{aligned}
& 2 \Delta\left(f_{0 t_{1}}-f_{0} f_{0 t_{1}}\right)=(\Delta+2) f_{0 t_{1} t_{1}}+\Delta^{2} \sum_{i=1}^{N}\left(\psi_{i} \phi_{i}^{(-1)}\right), \\
& \psi_{i, t_{1}}=\Delta\left(\psi_{i}\right)+f_{0} \psi_{i}, \phi_{i, t_{1}}=-\Delta^{*}\left(\phi_{i}\right)-f_{0} \phi_{i}, i=1,2, \cdots, N,
\end{aligned}
$$

which can be transformed to the second type of VSESCS.

\section{Dressing approach for exDKPH}

\subsection{Dressing approach for discrete KP hierarchy}

We first briefly recall the dressing approach for DKPH [11. Assume that operator $L$ of DKPH (4) can be written as a dressing form

$$
L=W \Delta W^{-1},
$$




$$
W=\Delta^{N}+w_{1} \Delta^{N-1}+w_{2} \Delta^{N-2}+\cdots+w_{N} .
$$

It is known [12] that if $W$ satisfies

$$
W_{t_{n}}=-L_{-}^{n} W
$$

then $L$ satisfies (44). It is easy to check the following Lemma.

Lemma 2. If $h_{t_{n}}=\Delta^{n}(h), W$ satisfies (24), then $\psi=W(h)$ satisfies (7), i.e.

$$
\psi_{t_{n}}=B_{n}(\psi) .
$$

If there are $N$ independent functions $h_{1}, \ldots, h_{N}$ solving $W(h)=0$ i.e. $W\left(h_{i}\right)=0$, then $w_{1}, \ldots, w_{N}$ are completely determined from these $h_{i}$, by solving the linear equation:

$$
\left(\begin{array}{cccc}
h_{1} & \Delta\left(h_{1}\right) & \cdots & \Delta^{N-1}\left(h_{1}\right) \\
h_{2} & \Delta\left(h_{2}\right) & \cdots & \Delta^{N-1}\left(h_{2}\right) \\
\vdots & \vdots & \vdots & \vdots \\
h_{N} & \Delta\left(h_{N}\right) & \cdots & \Delta^{N-1}\left(h_{N}\right)
\end{array}\right)\left(\begin{array}{c}
w_{N} \\
w_{N-1} \\
\vdots \\
w_{1}
\end{array}\right)=-\left(\begin{array}{c}
\Delta^{N}\left(h_{1}\right) \\
\Delta^{N}\left(h_{2}\right) \\
\vdots \\
\Delta^{N}\left(h_{N}\right)
\end{array}\right) .
$$

Then the operator $W$ can be written as

$$
W=\frac{1}{W r d\left(h_{1}, \cdots, h_{N}\right)}\left|\begin{array}{ccccc}
h_{1} & h_{2} & \cdots & h_{N} & 1 \\
\Delta\left(h_{1}\right) & \Delta\left(h_{2}\right) & \cdots & \Delta\left(h_{N}\right) & \Delta \\
\vdots & \vdots & \vdots & \vdots & \vdots \\
\Delta^{N}\left(h_{1}\right) & \Delta^{N}\left(h_{2}\right) & \cdots & \Delta^{N}\left(h_{N}\right) & \Delta^{N}
\end{array}\right|
$$

where $\operatorname{Wrd}\left(h_{1}, \cdots, h_{N}\right)=\left|\begin{array}{cccc}h_{1} & h_{2} & \cdots & h_{N} \\ \Delta\left(h_{1}\right) & \Delta\left(h_{2}\right) & \cdots & \Delta\left(h_{N}\right) \\ \vdots & \vdots & \vdots & \vdots \\ \Delta^{N-1}\left(h_{1}\right) & \Delta^{N-1}\left(h_{2}\right) & \cdots & \Delta^{N-1}\left(h_{N}\right)\end{array}\right|$.

Proposition 2. Assume that $h_{i}$ satisfies

$$
h_{i, t_{n}}=\Delta^{n}\left(h_{i}\right), i=1, \cdots, N
$$

$W$ and $L$ are constructed by (26) and (23), then $W$ and $L$ satisfy (24) and (4), respectively.

Proof. Taking partial derivative $\partial_{t_{n}}$ to the equation $W\left(h_{i}\right)=0$ :

$$
\begin{gathered}
W_{t_{n}}\left(h_{i}\right)+W \Delta^{n}\left(h_{i}\right)=\left(W_{t_{n}}+L_{+}^{n} W+L_{-}^{n} W\right)\left(h_{i}\right) \\
=\left(W_{t_{n}}+L_{-}^{n} W\right)\left(h_{i}\right)=0, i=1, \cdots, N,
\end{gathered}
$$

since $L_{-}^{n} W=L^{n} W-L_{+}^{n} W=W \Delta^{n}-L_{+}^{n} W, L_{-}^{n} W$ is a non-negative difference operator of order $<N, W_{t_{n}}+L_{-}^{n} W$ is also of order $<N$. Then according to the difference equation's theory, $W_{t_{n}}+L_{-}^{n} W$ is a zero operator. 


\subsection{Dressing approach for exDKPH}

We now generalized the dressing approach to exDKPH (9). We have

Lemma 3. Under (23), if $W$ satisfies 24) and

$$
W_{\tau_{k}}=-L_{-}^{k} W+\sum_{i=1}^{N} \psi_{i} \Delta^{-1} \phi_{i} W
$$

then $L$ satisfies (9a) and (9b).

Proof. It is known that $L$ satisfies (9a). We have

$$
\begin{aligned}
L_{\tau_{k}} & =W_{\tau_{k}} \Delta W^{-1}-W \Delta W^{-1} W_{\tau_{k}} W^{-1} \\
& =\left(-L_{-}^{k}+\sum_{i} \psi_{i} \Delta^{-1} \phi_{i}\right) L+L\left(L_{-}^{k}-\sum_{i} \psi_{i} \Delta^{-1} \phi_{i}\right)=\left[B_{k}+\sum_{i=1}^{N} \psi_{i} \Delta^{-1} \phi_{i}, L\right] .
\end{aligned}
$$

This dressing operator $W$ is constructed as follows: Let $g_{i}, \bar{g}_{i}$ satisfy

$$
\begin{array}{ll}
g_{i, t_{n}}=\Delta^{n}\left(g_{i}\right), & g_{i, \tau_{k}}=\Delta^{k}\left(g_{i}\right) \\
\bar{g}_{i, t_{n}}=\Delta^{n}\left(\bar{g}_{i}\right), & \bar{g}_{i, \tau_{k}}=\Delta^{k}\left(\bar{g}_{i}\right), i=1, \ldots, N .
\end{array}
$$

And let $h_{i}$ be the linear combination of $g_{i}$ and $\bar{g}_{i}$

$$
h_{i}=g_{i}+\alpha_{i}\left(\tau_{k}\right) \bar{g}_{i} \quad i=1, \ldots, N
$$

with the coefficient $\alpha_{i}$ being a differentiable function of $\tau_{k}$. Suppose $h_{1}, \ldots, h_{N}$ are still linearly independent.

Define

$$
\psi_{i}=-\dot{\alpha}_{i} W\left(\bar{g}_{i}\right), \phi_{i}=(-1)^{N-i} \frac{\operatorname{Wrd}\left(\Gamma h_{1}, \cdots, \hat{\Gamma h_{i}}, \cdots, \Gamma h_{N}\right)}{\operatorname{Wrd}\left(\Gamma h_{1}, \cdots, \Gamma h_{N}\right)}, i=1, \ldots, N
$$

where the hat ${ }^{\wedge}$ means rule out this term from the discrete Wronskian determinant, $\dot{\alpha}_{i}=\frac{d \alpha_{i}}{d \tau_{k}}$. We have

Proposition 3. Let $W$ be defined by (26) and (30), $L=W \Delta W^{-1}, \psi_{i}$ and $\phi_{i}$ be given by (31), then $W, L, \psi_{i}, \phi_{i}$ satisfy (24), (28) and exDKPH (9).

To prove it, we need several lemmas under the above assumptions. The first one is :

Lemma 4. (The discrete version of Oevel and Strampp's lemma [18])

$$
W^{-1}=\sum_{i=1}^{N} h_{i} \Delta^{-1} \phi_{i}
$$


Proof. Note that $\phi_{1}, \ldots, \phi_{N}$ defined in (31) satisfy the linear equation

$$
\sum_{i=1}^{N} \Delta^{j}\left(\Gamma h_{i}\right) \cdot \phi_{i}=\delta_{j, N-1}, \quad j=0,1, \cdots, N-1
$$

where $\delta_{j, N-1}$ is the Kronecker's delta symbol. Using properties $f \Delta^{-1}=$ $\sum_{j \geq 0} \Delta^{-j-1} \Delta^{j}(\Gamma f)$, we have

$$
\begin{aligned}
& \sum_{i=1}^{N} h_{i} \Delta^{-1} \phi_{i}=\sum_{i=1}^{N} \sum_{j=0}^{\infty} \Delta^{-j-1} \Delta^{j}\left(\Gamma\left(h_{i}\right)\right) \cdot \phi_{i}=\sum_{j=0}^{\infty} \Delta^{-j-1} \sum_{i=1}^{N} \Delta^{j}\left(\Gamma\left(h_{i}\right)\right) \cdot \phi_{i} \\
& =\sum_{j=0}^{N-1} \Delta^{-j-1} \delta_{j, N-1}+\sum_{j=N}^{\infty} \Delta^{-j-1} \sum_{i=1}^{N} \Delta^{j}\left(\Gamma\left(h_{i}\right)\right) \cdot \phi_{i}=\Delta^{-N}+O\left(\Delta^{-N-1}\right),
\end{aligned}
$$

So we have

$$
W \sum_{i} h_{i} \Delta^{-1} \phi_{i}=1+\left(W \sum_{i} h_{i} \Delta^{-1} \phi_{i}\right)_{-}=1+\sum_{i} W\left(h_{i}\right) \Delta^{-1} \phi_{i}=1 .
$$

This complete the proof.

Lemma 5. $W^{*}\left(\phi_{i}\right)=0$, for $i=1, \ldots, N$.

Proof. Lemma 1 implies that

$$
\left(\Delta^{-1} \phi_{i} W\right)_{-}=\Delta^{-1} W^{*}\left(\phi_{i}\right) .
$$

Using Lemma 4 and (10a), we have

$$
\begin{gathered}
0=\left(\Delta^{j} W^{-1} W\right)_{-1}=\left(\Delta^{j} \sum_{i=1}^{N} h_{i} \Delta^{-1} \phi_{i} W\right)_{-}=\left(\sum_{i=1}^{N} \Delta^{j}\left(h_{i}\right) \Delta^{-1} \phi_{i} W\right)_{-} \\
=\sum_{i=1}^{N} \Delta^{j}\left(h_{i}\right) \Delta^{-1} W^{*}\left(\phi_{i}\right), \quad j=0, \cdots, N-1 .
\end{gathered}
$$

Solving the equations with respect to $\Delta^{-1} W^{*}\left(\phi_{i}\right)$, we find $\Delta^{-1} W^{*}\left(\phi_{i}\right)=0$. This implies $W^{*}\left(\phi_{i}\right)=0$.

Lemma 6. The operator $\Delta^{-1} \phi_{i} W$ is a non-negative difference operator and

$$
\left(\Delta^{-1} \phi_{i} W\right)\left(h_{j}\right)=\delta_{i j}, 1 \leq i, j \leq N .
$$

Proof. Lemma 5 and (34) implies that $\Delta^{-1} \phi_{i} W$ is a non-negative difference operator. We define functions $c_{i j}=\left(\Delta^{-1} \phi_{i} W\right)\left(h_{j}\right)$, then $\Delta\left(c_{i j}\right)=\phi_{i} W\left(h_{j}\right)=$ 0 , which means $c_{i j}$ does not depend on the discrete variable $n$. From Lemma 4 we find that

$$
\sum_{i=1}^{N} \Delta^{k}\left(h_{i}\right) c_{i j}=\Delta^{k}\left(\sum_{i}\left(h_{i} \Delta^{-1} \phi_{i} W\right)\left(h_{j}\right)\right)=\Delta^{k}\left(W^{-1} W\right)\left(h_{j}\right)=\Delta^{k}\left(h_{j}\right),
$$

so $c_{i j}=\delta_{i j}$. 
Proof of Proposition 3. The proof of (24) is analogous to the proof of in the previous section. For (28), taking $\partial_{\tau_{k}}$ to the identity $W\left(h_{i}\right)=0$, using (29), (30), the definition (31) and Lemma 6, we find

$$
\begin{aligned}
0 & =\left(W_{\tau_{k}}\right)\left(h_{i}\right)+\left(W \Delta^{k}\right)\left(h_{i}\right)+\dot{\alpha}_{i} W\left(\bar{g}_{i}\right)=\left(W_{\tau_{k}}\right)\left(h_{i}\right)+\left(L^{k} W\right)\left(h_{i}\right)-\sum_{j=1}^{N} \psi_{j} \delta_{j i} \\
& =\left(W_{\tau_{k}}+L_{-}^{k} W-\sum_{j=1}^{N} \psi_{j} \Delta^{-1} \phi_{j} W\right)\left(h_{i}\right) .
\end{aligned}
$$

Since the non-negative difference operator acting on $h_{i}$ in the last expression has degree $<N$, it can not annihilate $N$ independent functions unless the operator itself vanishes. Hence (28) is proved. Then Lemma 3 leads to (9b). The first equation in (9c) is easy to be verified by a direct calculation, so it remains to prove the second equation in (9c). Firstly, we see that

$$
\left(W^{-1}\right)_{t_{n}}=-W^{-1} W_{t_{n}} W^{-1}=W^{-1}\left(L^{n}-B_{n}\right)=\Delta^{n} W^{-1}-W^{-1} B_{n} .
$$

Then we substitute $W^{-1}=\sum h_{i} \Delta^{-1} \phi_{i}$ to this equality at both ends, we have

$$
\begin{aligned}
& \left(W^{-1}\right)_{t_{n}}=\sum \Delta^{n}\left(h_{i}\right)^{-1} \phi_{i}+\sum h_{i} \Delta^{-1} \phi_{i, t_{n}} \\
& =\left(\Delta^{n} W^{-1}-W^{-1} B_{n}\right)_{-}=\sum \Delta^{n}\left(h_{i}\right) \Delta^{-1} \phi_{i}-\sum h_{i} \Delta^{-1} B_{n}^{*}\left(\phi_{i}\right)
\end{aligned}
$$

Then $\sum h_{i} \Delta^{-1} \phi_{i, t_{n}}=-\sum h_{i} \Delta^{-1} B_{n}^{*}\left(\phi_{i}\right)$ implies that (9c) holds.

\section{$5 \quad$ N-soliton solutions for exDKPH}

Using Proposition 3 we can find solutions to every equations in the exDKPH (9). Let us illustrate it by solving (13) and (15). For (13), let $\delta_{i}=e^{\lambda_{i}}-1$, $\kappa_{i}=e^{\mu_{i}}-1$, we take the solution of (29) as follows

$$
\begin{array}{r}
g_{i}:=\exp \left(l \lambda_{i}+\delta_{i} t_{1}+\delta_{i}^{2} \tau_{2}\right)=e^{\xi_{i}}, \quad \bar{g}_{i}:=\exp \left(l \mu_{i}+\kappa_{i} t_{1}+\kappa_{i}^{2} \tau_{2}\right)=e^{\eta_{i}} \\
h_{i}:=g_{i}+\alpha_{i}\left(\tau_{2}\right) \bar{g}_{i}=2 \sqrt{\alpha_{i}} \exp \left(\frac{\xi_{i}+\eta_{i}}{2}\right) \cosh \left(\Omega_{i}\right), \Omega_{i}=\frac{1}{2}\left(\xi_{i}-\eta_{i}-\ln \alpha_{i}\right) .
\end{array}
$$

Since $L=W \Delta W^{-1}=\Delta+f_{0}+f_{1} \Delta^{-1}+\cdots$, we have

$$
f_{0}=\operatorname{Res}_{\Delta}\left(W \Delta W^{-1} \Delta^{-1}\right)
$$

where $W$ is given by (26) and (36), then $f_{0}, \psi_{i}$ and $\phi_{i}$ given by (31) gives rise to the N-soliton solution for (13).

For example, we obtain 1-soliton solution for (13) with $N=1$ as follows

$$
f_{0}=\exp \left(\frac{\lambda_{1}+\mu_{1}}{2}\right)\left(\frac{\cosh \left(\Omega_{1}+2 \theta_{1}\right)}{\cosh \left(\Omega_{1}+\theta_{1}\right)}-\frac{\cosh \left(\Omega_{1}+\theta_{1}\right)}{\cosh \Omega_{1}}\right), \quad \theta_{1}=\frac{\lambda_{1}-\mu_{1}}{2}
$$




$$
\psi_{1}=-\frac{d \sqrt{\alpha_{1}}}{d \tau_{2}}\left(e^{\mu_{1}-\lambda_{1}}\right) \exp \frac{\xi_{1}+\eta_{1}}{2} \operatorname{sech} \Omega_{1}, \phi_{1}=\frac{e^{-\left(\lambda_{1}+\mu_{1}\right) / 2} \exp \left(-\frac{\xi_{1}+\eta_{1}}{2}\right)}{2 \sqrt{\alpha_{1}}} \operatorname{sech}\left(\Omega_{1}+\theta_{1}\right) .
$$

The 2-soliton solution of (13) with $N=2$ is given by

$$
\begin{aligned}
& f_{0}=-\Delta\left(w_{1}\right)=\left(e^{\lambda_{1}}+e^{\lambda_{2}}\right) \Delta\left(\frac{v_{1}}{v}\right), \\
& \psi_{1}=-\frac{\dot{\alpha}_{1}}{v}\left(1+\alpha_{2} \frac{\left(e^{\mu_{2}}-e^{\lambda_{1}}\right)\left(e^{\mu_{1}}-e^{\mu_{2}}\right)}{\left(e^{\lambda_{2}}-e^{\lambda_{1}}\right)\left(e^{\mu_{1}}-e^{\lambda_{2}}\right)} e^{\chi_{2}}\right)\left(e^{\mu_{1}}-e^{\lambda_{1}}\right)\left(e^{\mu_{1}}-e^{\lambda_{2}}\right) e^{\eta_{1}}, \\
& \psi_{2}=-\frac{\dot{\alpha}_{2}}{v}\left(1+\alpha_{1} \frac{\left(e^{\mu_{1}}-e^{\lambda_{2}}\right)\left(e^{\mu_{1}}-e^{\mu_{2}}\right)}{\left(e^{\lambda_{2}}-e^{\lambda_{1}}\right)\left(e^{\mu_{2}}-e^{\lambda_{1}}\right)} e^{\chi_{2}}\right)\left(e^{\mu_{2}}-e^{\lambda_{2}}\right)\left(e^{\mu_{2}}-e^{\lambda_{1}}\right) e^{\eta_{2}}, \\
& \phi_{1}=\Gamma\left(\frac{1+\alpha_{2} e^{\chi_{2}}}{\left(e^{\lambda_{1}}-e^{\lambda_{2}}\right) v} e^{-\xi_{1}}\right), \phi_{2}=\Gamma\left(\frac{1+\alpha_{1} e^{\chi_{1}}}{\left(e^{\lambda_{2}}-e^{\lambda_{1}}\right) v} e^{-\xi_{2}}\right),
\end{aligned}
$$

with

$$
\begin{gathered}
v=1+\alpha_{1} \frac{e^{\lambda_{2}}-e^{\mu_{1}}}{e^{\lambda_{2}}-e^{\lambda_{1}}} e^{\chi_{1}}+\alpha_{2} \frac{e^{\mu_{2}}-e^{\lambda_{1}}}{e^{\lambda_{2}}-e^{\lambda_{1}}} e^{\chi_{2}}+\alpha_{1} \alpha_{2} \frac{e^{\mu_{2}}-e^{\mu_{1}}}{e^{\lambda_{2}}-e^{\lambda_{1}}} e^{\chi_{1}+\chi_{2}}, \\
v_{1}=1+\alpha_{1} \frac{e^{2 \lambda_{2}}-e^{2 \mu_{1}}}{e^{\lambda_{2}}-e^{\lambda_{1}}} e^{\chi_{1}}+\alpha_{2} \frac{e^{2 \mu_{2}}-e^{2 \lambda_{1}}}{e^{\lambda_{2}}-e^{\lambda_{1}}} e^{\chi_{2}}+\alpha_{1} \alpha_{2} \frac{e^{2 \mu_{2}}-e^{2 \mu_{1}}}{e^{\lambda_{2}}-e^{\lambda_{1}}} e^{\chi_{1}+\chi_{2}} .
\end{gathered}
$$

It can be shown that the interaction between the two solutions is elastic.

For (15), we take the solution of (29) as follows

$$
\begin{gathered}
g_{i}:=\exp \left(l \lambda_{i}+\delta_{i} \tau_{1}+\delta_{i}^{2} t_{2}\right)=e^{\xi_{i}}, \quad \bar{g}_{i}:=\exp \left(l \mu_{i}+\kappa_{i} \tau_{1}+\kappa_{i}^{2} t_{2}\right)=e^{\eta_{i}} \\
h_{i}:=g_{i}+\alpha_{i}\left(\tau_{1}\right) \bar{g}_{i}=2 \sqrt{\alpha_{i}} \exp \left(\frac{\xi_{i}+\eta_{i}}{2}\right) \cosh \left(\Omega_{i}\right) .
\end{gathered}
$$

Then

$$
f_{0}=\operatorname{Res}_{\Delta}\left(W \Delta W^{-1} \Delta^{-1}\right), f_{1}=\operatorname{Res}_{\Delta}\left(W \Delta W^{-1}\right)
$$

together with $\psi_{i}$ and $\phi_{i}$ given by (31) presents the N-soliton solution for (15).

\section{Acknowledgement}

This work is supported by National Basic Research Program of China (973 Program) (2007CB814800), China Postdoctoral Science Foundation funded project (20080430420) and National Natural Science Foundation of China $(10801083,10671121)$.

\section{References}

[1] Date E, Jimbo M, Kashiwara M, and Miwa T 1981 J. Phys. Soc. Japan 50 3806.

[2] Van de Leur J W 1998 J. Math. Phys. 392833. 
[3] Kac V G and van de Leur J W 2003 J. Math. Phys. 443245.

[4] Mel'nikov V K 1987 Commun. Math. Phys. 112639.

[5] Liu X J 2008 Phys. Lett. A 3723819.

[6] Liu X J, Zeng Y B and Lin R L 2008 J. Math. Phys. 49093506.

[7] Lin R L, Liu X J and Zeng Y B A new extended q-deformed KP hierarchy, to appear in J. Nonlinear Math. Phys.

[8] Wu H X, Liu X J and Zeng Y B 2008 J. Math. Phys. 49093510.

[9] Date E, Jimbo M and Miwa T 1982 Phys. Soc. Jpn. 51 4116,4125.

[10] Oevel W Darboux transformation for integrable lattice system, Nonlinear Physics. 1996 Theory and Experiment, 233-240, E. Alfinito, L.Martina and F. Pempinelli(eds), World Scientific, Singapore.

[11] Vel Kanaga S and Tamizhmani K M 1997 Chaos, Solitons and Fractals 8 917.

[12] Dickey L A 2003 Soliton equations and Hamiltonian systems, World Scientific, Singapore.

[13] Adeler V E, Bobenko A I and Suris Yu B 2003 Comm. Math. Phys. 233 513.

[14] Date E, Kashiwava M, Jimbo M and Miwa T 1983 "Transformation groups for soliton equations" in Nonlinear integrable system-classical theory and quantum theory. World Scientific, Singapore, 39-119

[15] Chau L L, Shaw J C and Yen H C 1992 Comm. Math. Phys. 149263.

[16] He J S, Liu S W and Cheng Y The determinant representation of the gauge transformation for discrete KP hierarchy, preparation.

[17] Liu X J, Lin R L and Zeng Y B A generalized dressing approach for solving the extended KP and the extended mKP Hierarchy, preparation.

[18] Oevel W and Strampp W 1996 J. Math. Phys. 376213.

[19] Veselov A P and Shabat A B 1993 Funct. Anal. Appn. 2781. 